\title{
Prophylactic Venous Supercharged Radial Collateral Artery Perforator Propeller Flap: Improved Outcome in Perforator Propeller Flaps
}

\author{
Damir Kosutic, MD, PhD, FRCS (Plast) ${ }^{1}$ Pratap Dutta, MBBS, MS, MRCSEd ${ }^{1}$ \\ Ingrid Kieran, $\mathrm{MD}, \mathrm{MRCS}, \mathrm{MB}, \mathrm{BCh}, \mathrm{BAO}^{1}$
}

${ }^{1}$ Department of Plastic Surgery, The Christie NHS Foundation Trust, Manchester, United Kingdom

J Reconstr Microsurg Open 2016;1:45-47.

Address for correspondence Damir Kosutic, MD, PhD, FRCS (Plast), Department of Plastic Surgery, The Christie NHS Foundation Trust, Wilmslow Road, M20 4BX, Manchester, United Kingdom (e-mail: dkosutic@hotmail.com).

The flap was rotated 180 degrees on this single perforator and set into the defect. The flap remained largely well perfused; however, there were signs of congestion at the distal end of the flap. The dissected superficial vein in the flap was therefore anastomosed to the recipient superficial vein in the forearm defect (-Fig. 2A). The congestion initially observed rapidly dissipated several minutes after the venous anastomosis. Excellent blood flow was observed.

The donor site was closed directly. Postoperative wound healing was uneventful and the entire flap survived. Full range of movement was maintained at the elbow, and the patient had an excellent aesthetic outcome. This was observed by surgeon and reported by the patient in terms of a highly satisfied outcome (- Fig. 2B).

\section{Case 2}

A 73-year-old woman underwent a 2-cm-wide local excision of a recurrent melanoma on her right arm. The subsequent defect measured $12 \times 9 \mathrm{~cm}$. Using a Doppler, an RCAP propeller flap was designed on the dorsal proximal forearm around a perforator located in close proximity to the lateral humeral epicondyle ( $\mathbf{- F i g . ~ 3 A )}$ ). The flap was raised and an additional superficial vein was dissected for prophylactic augmentation of venous drainage. The flap was rotated 130 degrees counterclockwise into the defect and the superficial vein on its tip was anastomosed to a branch of the cephalic vein (-Fig. 3B). No venous congestion was observed.

The donor site was closed directly. Postoperative wound healing was uneventful, the entire flap survived, and full range of movement of the elbow was maintained ( - Fig. $3 \mathbf{C}$ ). received

April 26, 2015

accepted after revision

August 23, 2015

published online

February 11, 2016
DOI http://dx.doi.org/

10.1055/s-0035-1570533. ISSN 2377-0813.
Copyright $\odot 2016$ by Thieme Medical Publishers, Inc., 333 Seventh Avenue, New York, NY 10001, USA. Tel: +1(212) 584-4662.
License terms

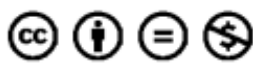




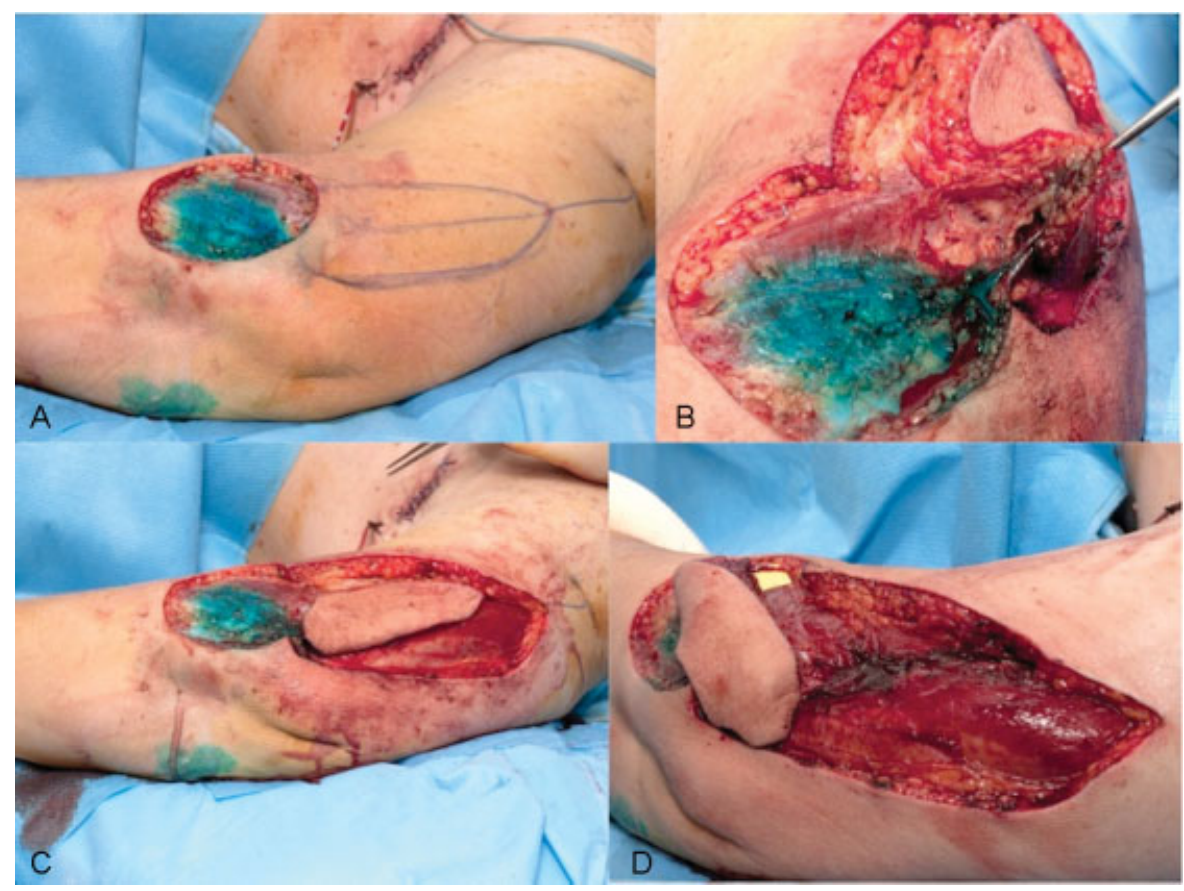

Fig. 1 A-D Left upper arm RCAP propeller flap designed around the perforator in close proximity to lateral epicondyle and harvested from upper arm.

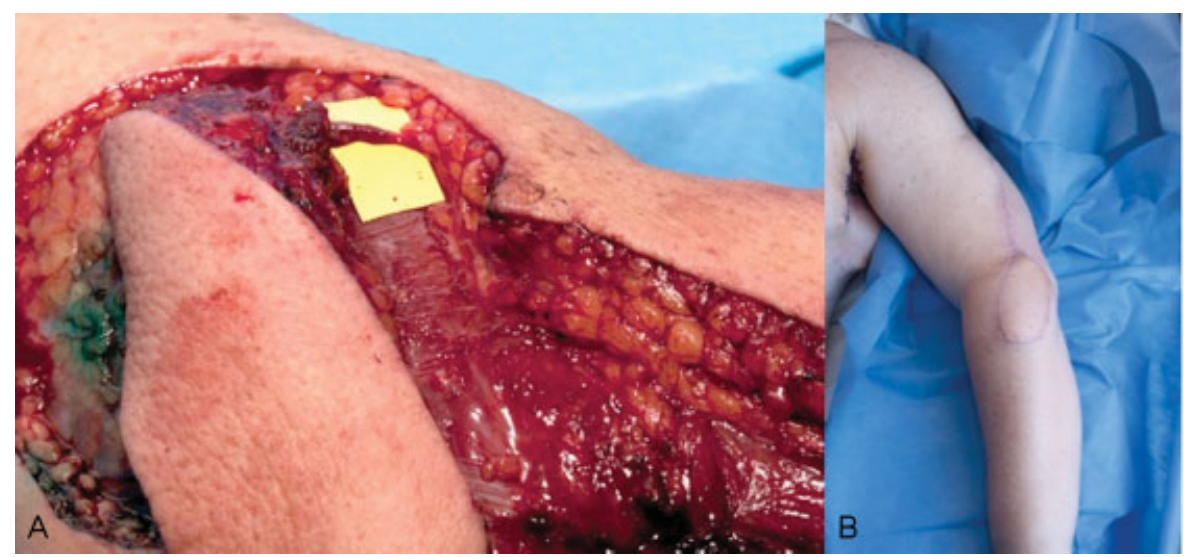

Fig. 2 A Prophylactic venous supercharging of RCAP flap by anastomosing superficial vein at the tip of the flap with superficial vein on the dorsal forearm following flap rotation. B Excellent functional and aesthetic outcome at 6-month follow-up.

\section{Discussion}

Favorable characteristics of the RCAP propeller flap include a reliable perforator, ease of dissection, and avoidance of sacrificing major pedicles and muscle in a single-stage reconstructive procedure for small- to medium-sized defects, ${ }^{2}$ including both skin and the bone. ${ }^{3}$ However, as with all propeller flaps, there is a risk of venous congestion following rotation and flap inset. This can delay wound healing, entail further surgical procedures, and negatively impact final overall function and aesthetics. ${ }^{1}$ This undermines the benefits of the propeller flap technique which can be used as a reliable, one-stage, and aesthetically pleasing reconstructive technique. The time taken for dissection of this superficial vein within the distal end of the flap, as well as the recipient vessel, is minimal with an abundance of veins of adequate caliber available in the dorsal forearm and arm.

These cases illustrate that with inclusion of an additional superficial vein for augmenting venous drainage, larger flaps can safely be raised and the risk of venous congestion as well as flap necrosis can be reduced. This also introduces the possibility of performing this more robust technique safely as a day case eliminating the need for hospital 


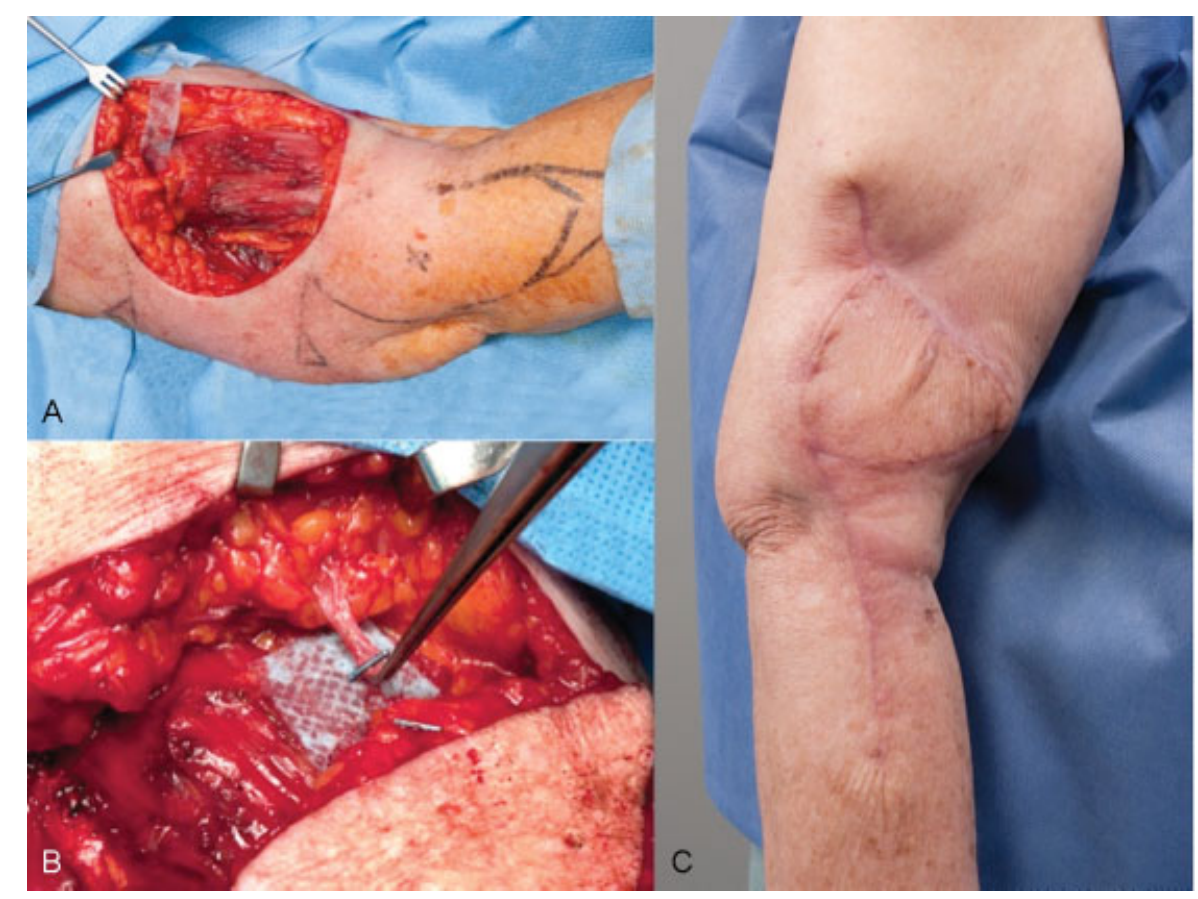

Fig. 3 A, B Forearm RCAP flap for reconstruction of upper arm defect based on perforator next to lateral epicondyle, rotated into upper arm defect. Branch of cephalic vein dissected as a recipient for venous supercharging. C Flap healed with full range of motion in elbow on follow-up.

admission and flap observations. We propose the prophylactically venous supercharged RCAP propeller flap as a safe and reliable option in reconstructing challenging defects in the arm, forearm, and elbow region.

\section{Acknowledgment}

The authors thank the Medical Illustration Department at Christie Hospital for preparation of excellent quality clinical photographs.

\section{References}

1 Innocenti M, Menichini G, Baldrighi C, Delcroix L, Vignini L, Tos P. Are there risk factors for complications of perforator-based propeller flaps for lower-extremity reconstruction? Clin Orthop Relat Res 2014;472(7):2276-2286

2 Murakami M, Ono S, Ishii N, Hyakusoku H. Reconstruction of elbow region defects using radial collateral artery perforator (RCAP)-based propeller flaps. J Plast Reconstr Aesthet Surg 2012;65(10):1418-1421

3 Song D, Xu J, Lv H, Liu J, Li J. Wraparound chimeric radial collateral artery perforator flap for reconstruction of thumb loss. J Reconstr Microsurg 2015;31(2):95-101 